\title{
Marcação de fitomassa de cana-de-açúcar com aplicação de solução de uréia marcada com ${ }^{15} \mathrm{~N}$
}

\author{
Carlos Eduardo Faroni( ${ }^{(1)}$, Paulo Cesar Ocheuze Trivelin(2), Priscila Helena da Silva(1), \\ Isabela Rodrigues Bologna ${ }^{(1)}$, André César Vitti(3) e Henrique Coutinho Junqueira Franco ${ }^{(1)}$
}

\begin{abstract}
(1)Escola Superior de Agricultura Luiz de Queiroz, Caixa Postal 9, CEP 13418-900 Piracicaba, SP. E-mail: cefaroni@esalq.usp.br; priscila_helena@yahoo.com.br, irbologn@esalq.usp.br, hjfranco@cena.usp.br (2)Centro de Energia Nuclear na Agricultura, Laboratório de Isótopos Estáveis, Caixa Postal 96, CEP 13400-970 Piracicaba, SP. E-mail: pcotrive@cena.usp.br (3)Apta - Pólo Regional Centro Sul, Caixa Postal 128, CEP 13400-970 Piracicaba, SP. E-mail: acvitti@aptaregional.sp.gov.br
\end{abstract}

\begin{abstract}
Resumo - O objetivo deste trabalho foi comparar três métodos de aplicação de solução de uréia marcada com ${ }^{15} \mathrm{~N}\left({ }^{15} \mathrm{~N}\right.$-uréia ): pulverização foliar, injeção na base do colmo e imersão radicular, a fim de se definir qual seria o mais eficiente na marcação de fitomassa de cana-de-açúcar. O experimento foi instalado na Estação Experimental Apta - Pólo Regional Centro Sul, em Piracicaba, SP. A cana-de-açúcar, variedade SP80 3280, foi plantada em vasos preenchidos com aproximadamente $120 \mathrm{dm}^{3}$ de Neossolo Quartzarênico de textura arenosa. O delineamento experimental foi inteiramente casualizado, com quatro repetições. A fitomassa de cana-de-açúcar dos três tratamentos, no $11^{\circ}$ mês de desenvolvimento, não diferiu estatisticamente, e suas abundâncias de ${ }^{15} \mathrm{~N}$ foram superiores à natural, tendo-se verificado a seguinte ordem decrescente de marcação com ${ }^{15} \mathrm{~N}$ : parte aérea > rizoma > rizomas+raízes na camada de $0,0-0,2 \mathrm{~m}>$ raízes na camada de $0,2-0,4 \mathrm{~m}>$ raízes em profundidade maior que $0,4 \mathrm{~m}$. Entre os métodos de aplicação de ${ }^{15} \mathrm{~N}$-uréia, a injeção na base de colmos é o de mais fácil execução, o mais efetivo na marcação da fitomassa e o que apresentou a maior recuperação do traçador (96\%). A aplicação foliar é comparável à injeção, somente na marcação e na recuperação do traçador no sistema radicular.
\end{abstract}

Termos para indexação: Saccharum spp., parte aérea, sistema radicular, marcação isotópica.

\section{Sugarcane phytomass labeling with application of ${ }^{15} \mathrm{~N}$-urea solution}

\begin{abstract}
The objective of this research was to compare three methods of ${ }^{15} \mathrm{~N}$-urea solutions application: spray on leaf, injection in the plant base stem and root immersion, in order to define the most efficient labeling sugarcane phytomass with ${ }^{15} \mathrm{~N}$ method. The experiment was carried out at APTA - Pólo Regional Centro Sul, in Piracicaba, SP, Brazil, and the sugarcane variety SP80 3280 was planted in pots filled out with approximately $120 \mathrm{dm}^{3}$ of a Typic Quartzipsamment soil. The experiment was conducted in a completely randomized design with four replicates. There were no difference between the methods in the plant parts dry mass, in $11^{\circ}$ development month, and their ${ }^{15} \mathrm{~N}$ abundances were superior to the natural one, being verified the following decreasing labeling order: aerial part $>$ rhizome $>$ rhizome + roots in the layer $0.0-0.2 \mathrm{~m}>$ roots in the layer $0.2-0.4 \mathrm{~m}>$ roots in the layer deeper than $0.4 \mathrm{~m}$. Among the ${ }^{15} \mathrm{~N}$-urea solution application methods, the injection in the base stem is the easiest to apply, the most effective in dry mass labeling and the one wich presented the largest ${ }^{15} \mathrm{~N}$ tracer recovery (96\%). The spray on leaf method is comparable to the injection in the base stem only for the labeling with ${ }^{15} \mathrm{~N}$ and tracer recovery for root system.
\end{abstract}

Index terms: Saccharum spp., aerial part, root system, isotope labeling.

\section{Introdução}

A produção de fitomassa pelas culturas é um dos parâmetros mais avaliados na experimentação agronômica. Subentende-se por fitomassa da cana-de-açúcar todo o material vegetal da parte aérea e do sistema radicular (rizomas e raízes); este último é essencial à regeneração da cana-de-açúcar após as colheitas, pois cumpre papel de reserva orgânica e nutricional para a futura rebrota da cultura (Trivelin et al., 2002).

As maiores limitações ambientais à produção de fitomassa pela cana-de-açúcar no Brasil, se relacionam à disponibilidade de quantidades adequadas de nutrientes minerais nos solos, com destaque ao $\mathrm{N}$ (Trivelin et al., 2002). Esse nutriente pode ter seus efeitos estudados com o uso de compostos marcados no isótopo estável 
${ }^{15} \mathrm{~N}$, na quantificação das taxas de transferência do nutriente para diferentes compartimentos do agrossistema (Trivelin, 2000). A marcação da fitomassa com ${ }^{15} \mathrm{~N}$ pode ser realizada com diferentes objetivos, como por exemplo, avaliar a mineralização do $\mathrm{N}$ dos restos culturais e sua contribuição à nutrição da cultura (Gava et al., 2005), como também, o desenvolvimento e a distribuição de raízes metabolicamente ativas (Faroni $\&$ Trivelin, 2006).

A aplicação foliar com ${ }^{15} \mathrm{~N}$-uréia é um dos métodos de marcação mais empregados em estudos sobre nutrição nitrogenada, em várias culturas, e sobre a fixação de $\mathrm{N}_{2}$ por leguminosas (Wittwer et al., 1963; McNeill et al., 1997; Ambrosano et al., 2003), mas é desuniforme quanto à distribuição do isótopo nas partes das plantas, embora seja de fácil execução (Zebarth et al., 1991). Em levantamento de técnicas de marcação de fitomassa com ${ }^{15} \mathrm{~N}$, realizado por Russel \& Fillery (1996), a aplicação foliar com ${ }^{15} \mathrm{~N}$-uréia, apesar de ser o método de marcação mais utilizado, não foi satisfatório para o estudo de transferência de $\mathrm{N}$ no sistema, por não poder ser feito um balanço total do N. Uma característica desse método é a perda de $\mathrm{NH}_{3}$ pela hidrólise da uréia ou por ação da chuva ou do orvalho, que podem transferir o ${ }^{15} \mathrm{~N}$ ao solo o que invalida a estimativa da quantidade de ${ }^{15} \mathrm{~N}$ nesse compartimento (Vasilas et al., 1980).

$\mathrm{Na}$ tentativa de contornar os inconvenientes da aplicação foliar, outros métodos foram desenvolvidos, como a imersão de raízes em uma solução com fontes nitrogenadas marcadas com ${ }^{15} \mathrm{~N}$, técnica que supera muito dos problemas inerentes às aplicações foliares, mas que também é destrutiva, quando o objeto do estudo é o sistema radicular, já que se torna necessária a exposição e o corte ou fendilhamento de raízes, para sua posterior imersão em solução com o isótopo. No entanto, o sistema confinado propicia uma grande absorção de ${ }^{15} \mathrm{~N}$ pela eliminação das perdas externas. Outra técnica que pode ser usada é a injeção de solução com fontes marcadas com ${ }^{15} \mathrm{~N}$, a qual tem sido empregada com sucesso em leguminosas arbustivas, com a ressalva de que, para ser bem sucedida, deve ser empregada em plantas maduras, por causa da dependência de maior diâmetro de caule que suporte a quantidade e a concentração da solução a ser injetada (Russel \& Fillery, 1996).

Os métodos descritos apresentam vantagens e desvantagens, a depender da planta de interesse, mas em todos eles a uréia apresenta-se como uma fonte de $\mathrm{N}$ adequada para fornecimento do marcador isotópico
${ }^{15} \mathrm{~N}$, pela sua solubilidade em água e alto conteúdo de $\mathrm{N}$, o que permite aplicações de boa quantidade do nutriente, quer seja aplicado em solução ou na forma sólida (Thorne, 1955; Wittwer \& Teubner, 1959). Conforme Trivelin et al. (1988), o N aplicado às folhas de cana-de-açúcar e translocado às raízes indicou efeito estatístico para o fator tempo, o que evidencia que a translocação de $\mathrm{N}$ foi contínua até 96 horas após a fertilização foliar, e a colheita da fitomassa deve ser realizada somente após esse período.

O objetivo deste trabalho foi o de comparar os métodos: pulverização foliar, imersão radicular e injeção na base do colmo, a fim de se definir qual o mais eficiente na marcação da fitomassa de cana-de-açúcar.

\section{Material e Métodos}

O experimento foi instalado na Estação Experimental Apta - Pólo Regional Centro Sul, em Piracicaba, SP. A cana-de-açúcar foi plantada em vasos com volume aproximado de $120 \mathrm{dm}^{3}$, preenchidos com Neossolo Quartzarênico de textura arenosa (Embrapa, 1999). Os vasos continham dreno de brita zero, de $0,1 \mathrm{~m}$ de altura, coberto com manta do tipo Bidim. Após a análise química da terra, foi realizada a correção de sua acidez, em cada vaso, com a aplicação de $93,04 \mathrm{~g}$ de $\mathrm{CaCO}_{3}$ para elevar a saturação de bases a 70\% (Raij, 1991). Foi realizada, também, a fosfatagem da terra segundo Espironelo (1996), corrigida para estudos em vasos, com a aplicação de 94,38 g de superfosfato simples à terra de cada vaso.

A cultivar de cana-de-açúcar utilizada foi a SP80 3280, de maturação média, alta produtividade e exigência média em fertilidade do solo. Foram plantados três minitoletes por vaso, com uma gema cada que, depois da brotação, foram selecionados, tendo permanecido apenas um perfilho primário por vaso (parcela). Cada parcela recebeu doses de uréia, equivalente a $50 \mathrm{~kg} \mathrm{ha}^{-1}$ de $\mathrm{N}$, e de cloreto de potássio, equivalente a $75 \mathrm{~kg} \mathrm{ha}^{-1}$ de $\mathrm{K}_{2} \mathrm{O}$. Durante o crescimento das plantas, foram realizadas adubações de cobertura, com doses idênticas às do plantio: a primeira com ambos os nutrientes, e a segunda apenas com $\mathrm{N}$, tendo sido o $\mathrm{N}$ aplicado nas formas de uréia e sulfato de amônio, respectivamente, no plantio e cobertura. Foram realizadas irrigações para suprir a necessidade hídrica.

Os métodos de aplicação do traçador foram: pulverização foliar, injeção no colmo da planta e imersão radicular. $\mathrm{O}$ delineamento experimental foi o inteiramente 
casualizado, com 4 repetições. A uréia marcada com ${ }^{15} \mathrm{~N}$ ( ${ }^{15} \mathrm{~N}$-uréia) foi utilizada para a marcação das plantas, quando estas estavam no 11으ês de desenvolvimento, e sua abundância isotópica foi maior quanto menor o volume de solução administrado à planta (Tabela 1).

A pulverização foliar foi feita por meio de um pulverizador (spray), tendo-se aplicado $16 \mathrm{~mL}$ de solução de uréia, com abundância de 15,27\% em átomos de ${ }^{15} \mathrm{~N}$, às folhas e às bainhas das plantas; neste método, o solo foi coberto com lona de plástico, para impedir sua possível marcação por deriva ou escorrimento da solução aplicada. A injeção foi feita por meio de uma seringa de $1 \mathrm{~mL}$ e agulha de dimensões $0,45 \times 13 \mathrm{~mm}$, aplicada à base dos colmos das plantas, no total de $3 \mathrm{~mL}$ de solução de uréia, com abundância de 81,42\% em átomos de ${ }^{15} \mathrm{~N}$. A absorção radicular foi feita pela imersão de raízes, que evidenciavam, por inspeção visual, apresentar metabolismo ativo, em frascos tipo Ependorf, que continham solução idêntica em volume, concentração e abundância isotópica à do método de injeção no colmo das plantas. Nos dois últimos métodos mencionados, o volume de $3 \mathrm{~mL}$ de solução, aplicado ou fornecido à planta, foi dividido entre os colmos de cada parcela, em quantidade não superior a $0,5 \mathrm{~mL}$ por colmo, perfazendo, no mínimo, 6 aplicações por vaso.

A colheita do experimento ocorreu após 96 horas da aplicação do traçador (Trivelin, 1988; Faroni \& Trivelin, 2006). A parte aérea (colmo+ponteiros) de cada parcela foi colhida, pesada e triturada em um desfibrador tipo forrageira. Da fitomassa triturada foi retirada uma subamostra, que foi secada em estufa de circulação forçada de ar, a $65^{\circ} \mathrm{C}$, até atingir massa constante, para determinação da fitomassa seca da parte aérea. Nessa subamostra, foram realizadas as determinações de concentração de $\mathrm{N}$ e de abundância de ${ }^{15} \mathrm{~N}$.

A colheita do sistema radicular foi feita às profundidades de $0-0,2 \mathrm{~m}$, de $0,2-0,4 \mathrm{~m}$ e abaixo de $0,4 \mathrm{~m}$, tendo-se separado os rizomas e as raízes do solo com uso de peneira de $2 \mathrm{~mm}$ de malha, os quais foram lavados em água corrente, para a retirada do excesso da terra aderida. Foi quantificada a massa dos rizomas e raízes após secagem em estufa a $65^{\circ} \mathrm{C}$, e determinada a concentração de $\mathrm{N}$ e a abundância isotópica de ${ }^{15} \mathrm{~N}$.

As determinações da concentração de $\mathrm{N}$ e de ${ }^{15} \mathrm{~N}$, nas amostras, foram realizadas em espectrômetro de massas automatizado, modelo ANCA SL 20-20 (Barrier \& Prosser, 1996). A recuperação do marcador aplicado foi calculada pela fórmula: Recuperação $(\%)=100\left(\mathrm{NPPM} / \mathrm{M}^{15 \mathrm{~N}} \mathrm{U}\right)$, em que: NPPM é o $\mathrm{N}$ da planta proveniente do marcador ${ }^{15} \mathrm{~N}$-uréia; $\mathrm{M}^{15 \mathrm{~N}} \mathrm{U}$ é a massa de $\mathrm{N}$ do marcador isotópico ${ }^{15} \mathrm{~N}$-uréia aplicado por parcela. NPPM $=\left(\%{ }^{15} \mathrm{~N}_{\text {exc. compartimento }} /\right.$ $\left.\%{ }^{15} \mathrm{~N}_{\text {exc. marcador }}\right) \times \mathrm{N}$-total, em que: $\%{ }^{15} \mathrm{~N}_{\text {exc. compartimento }} \mathrm{e}$ $\%{ }^{15} \mathrm{~N}_{\text {exc marcador }}$ representam a porcentagem de átomos de ${ }^{15} \mathrm{~N}$ em excesso no compartimento e no marcador, respectivamente; a abundância natural em átomos de ${ }^{15} \mathrm{~N}$ é igual a $0,367 \%$; $\mathrm{N}$-total ${ }_{\mathrm{c}}$ é a massa de $\mathrm{N}$ total no compartimento.

Os resultados de massa de matéria seca, de abundância e de recuperação de ${ }^{15} \mathrm{~N}$, por compartimento das plantas, foram analisados por meio de análise de variância e, naqueles em que houve diferença significativa, os dados foram submetidos ao teste de Tukey, a $5 \%$ de probabilidade.

\section{Resultados e Discussão}

Em relação à aplicabilidade dos métodos, a pulverização foliar foi de fácil e rápida aplicação, mesmo com ressalvas quanto a alguns pontos a serem considerados. O volume de solução aplicado deve ser proporcional à área foliar e à capacidade de retenção da solução pela superfície da folha, a fim de se evitarem o escorrimento e a perda da solução com ${ }^{15} \mathrm{~N}$. Quanto menor o volume de solução a aplicar, maior deverá ser a abundância de ${ }^{15} \mathrm{~N}$, para que a massa do traçador seja adequada à marcação desejada da fitomassa, que deve ser calculada em função da fitomassa seca total da planta e de seu teor de $\mathrm{N}$ no estádio de colheita.

Tabela 1. Massas de uréia, $\mathrm{N}$ e ${ }^{15} \mathrm{~N}$ aplicadas por parcela (vaso), em cada tratamento, e seus respectivos volumes e concentrações ${ }^{(1)}$.

\begin{tabular}{|c|c|c|c|c|c|c|}
\hline Método de marcação & $\begin{array}{c}\text { Massa de } \\
\text { uréia }\end{array}$ & $\begin{array}{c}\text { Massa de } \\
\mathrm{N}^{(1)}\end{array}$ & $\begin{array}{l}\text { Volume de } \\
\text { solução }\end{array}$ & Concentração & $\begin{array}{c}\text { Abundância de }{ }^{15} \mathrm{~N} \\
\text { na uréia }\end{array}$ & Massa de ${ }^{15} \mathrm{~N}$ \\
\hline & \multicolumn{2}{|c|}{-------(g por vaso)------- } & (mL por vaso) & $(\%(\mathrm{~m} / \mathrm{v}))$ & (átomos\%) & (g por vaso) \\
\hline Pulverização foliar & $3,26^{\circ}$ & 1,538 & 16 & 20 & 15,27 & 0,235 \\
\hline Injeção no colmo da planta & 0,61 & 0,293 & 6 & 10 & 81,42 & 0,239 \\
\hline Imersão radicular & 0,61 & 0,293 & 6 & 10 & 81,42 & 0,239 \\
\hline
\end{tabular}

${ }^{(1)} \mathrm{O}$ peso atômico do $\mathrm{N}$ foi de 14,16 e 14,81 , respectivamente, para a uréia com abundância de 15,27 e $81,42 \%$ em átomos de ${ }^{15} \mathrm{~N}$. 
No caso de plantas com perfilhamento, como a canade-açúcar, a aplicação deve ser realizada nas folhas de todos os perfilhos, para que a distribuição pela planta seja a mais uniforme possível, principalmente no caso de cana-soca, em que cada perfilho possui um sistema radicular próprio. A principal desvantagem do método é o tempo necessário para absorção da solução aplicada às folhas (Malavolta, 1994), a qual não é totalmente absorvida e pode, em parte, ser perdida na forma de $\mathrm{NH}_{3}$, após sua hidrólise por ação da urease celular (Witte et al., 2002). A permanência, sobre as folhas, de resíduos de uréia na forma de cristais desidratados (Humbert, 1960) possibilita que a chuva, o orvalho ou a irrigação promova a remoção de parte da uréia aplicada e carregue o traçador ${ }^{15} \mathrm{~N}$ para o solo.

A injeção da solução com ${ }^{15} \mathrm{~N}$-uréia no colmo da planta foi de fácil realização, da mesma forma como a pulverização foliar. A vantagem que pode ser destacada neste método é a de que toda solução pode ser aplicada diretamente à planta, e isso garante que não ocorra perda de ${ }^{15} \mathrm{~N}$ por volatilização de $\mathrm{NH}_{3}$, como na aplicação foliar. Assim, pode ser realizado o balanço efetivo de ${ }^{15} \mathrm{~N}$ na planta e solo. Como desvantagem do método, pode-se citar o reduzido volume a ser aplicado, o que exige que a abundância do isótopo seja tanto maior, quanto maior for a quantidade da fitomassa seca a ser marcada. Para esse método, a aplicação do marcador deve ser feita em todos os perfilhos, a fim de que toda touceira possa ser marcada.

O método da imersão radicular, com poucas informações na literatura (Russel \& Fillery, 1996), também foi usado com certa facilidade, no entanto, com um tempo para aplicação bem superior aos demais. Este método foi o que apresentou as maiores desvantagens, como a exposição das raízes, as quais foram imersas na solução com o marcador, o que causou perturbação no sistema colhido, como a perda de raízes. Outra desvantagem foi que nem toda solução contida nos frascos foi absorvida.

Não houve diferença significativa, entre os métodos de marcação (Tabela 2), na comparação das médias das massas de matéria seca da parte aérea, dos rizomas e das raízes de $0,0-0,2 \mathrm{~m}, 0,2-0,4 \mathrm{~m}$ e $>0,4 \mathrm{~m}$, e a probabilidade de assimilação do marcador isotópico foi a mesma para todos os tratamentos. Esse resultado é fundamental, neste tipo de estudo, pois como foi fornecida uma mesma massa de ${ }^{15} \mathrm{~N}$ a todas as parcelas do experimento (Tabela 1), se uma dessas apresentasse maior massa ocorreria maior diluição isotópica e vice-versa.

A abundância de ${ }^{15} \mathrm{~N}$, de todas as partes das plantas, foi superior à abundância natural do solo $(0,367 \%$ em átomos de ${ }^{15} \mathrm{~N}$ ), tendo-se verificado a seguinte ordem decrescente de marcação: parte aérea $>$ rizoma $>$ raízes na camada de $0,0-0,2 \mathrm{~m}>$ raízes na camada de $0,2-0,4 \mathrm{~m}$ $>$ raízes na profundidade maior que $0,4 \mathrm{~m}$ (Tabela 3 ).

A marcação da planta com ${ }^{15} \mathrm{~N}$ (Tabela 3 ) foi diferente nas partes ou compartimentos das plantas, conforme o método de marcação. Para a marcação da parte aérea, houve diferença significativa $(p>0,01)$ entre os tratamentos; o método da injeção no colmo das plantas

Tabela 2. Massa de matéria seca (g por vaso) da parte aérea, rizomas e raízes das plantas com diferentes métodos de marcação.

\begin{tabular}{|c|c|c|c|c|c|}
\hline \multirow[t]{2}{*}{ Método de marcação } & \multirow[t]{2}{*}{ Parte aérea } & \multirow[t]{2}{*}{ Rizomas } & \multicolumn{3}{|c|}{ Raízes } \\
\hline & & & $0,0-0,2 \mathrm{~m}$ & $0,2-0,4 \mathrm{~m}$ & $>0,4 \mathrm{~m}$ \\
\hline Pulverização foliar & 1.401 & 280 & 86 & 32 & 68 \\
\hline Injeção no colmo & 1.474 & 281 & 83 & 36 & 57 \\
\hline Absorção radicular & 1.382 & 351 & 73 & 32 & 71 \\
\hline Valor de $\mathrm{F}$ & $0,14^{\mathrm{ns}}$ & $0,99^{\mathrm{ns}}$ & $0,42^{\mathrm{ns}}$ & $0,90^{\mathrm{ns}}$ & $0,43^{\text {ns }}$ \\
\hline $\mathrm{CV}(\%)$ & 19 & 27 & 26 & 17 & 35 \\
\hline
\end{tabular}

${ }_{\text {ns Não-significativo. }}$

Tabela 3. Abundância de ${ }^{15} \mathrm{~N}\left(\%\right.$ em átomos de $\left.{ }^{15} \mathrm{~N}\right)$ na parte aérea, rizomas e raízes das plantas com diferentes métodos de marcação ${ }^{(1)}$.

\begin{tabular}{|c|c|c|c|c|c|}
\hline \multirow[t]{2}{*}{ Método de marcação } & \multirow[t]{2}{*}{ Parte aérea } & \multirow[t]{2}{*}{ Rizomas } & \multicolumn{3}{|c|}{ Raízes } \\
\hline & & & $0,0-0,2 \mathrm{~m}$ & $0,2-0,4 \mathrm{~m}$ & $>0,4 \mathrm{~m}$ \\
\hline Pulverização foliar & $2,051 \mathrm{~b}$ & 2,081 & 1,470 & $0,675 \mathrm{ab}$ & $0,537 \mathrm{ab}$ \\
\hline Injeção no colmo & $4,418 \mathrm{a}$ & 2,333 & 1,441 & $0,826 a$ & $0,613 a$ \\
\hline Absorção radicular & $1,295 b$ & 1,204 & 1,095 & $0,512 b$ & $0,453 \mathrm{~b}$ \\
\hline Valor de $\mathrm{F}$ & $36,50 * *$ & $2,45^{\text {ns }}$ & $1,54^{\mathrm{ns}}$ & $5,40^{*}$ & $4,51^{*}$ \\
\hline $\mathrm{CV}(\%)$ & 22 & 40 & 23 & 19 & 14 \\
\hline
\end{tabular}

${ }^{(1)}$ Médias seguidas por letras iguais, na coluna, não diferem entre si pelo teste de Tukey, a $5 \%$ de probabilidade. ${ }^{\text {ns Não-significativo. } * \text { e } * * \text { Significativo }}$ a 5 e $1 \%$ de probabilidade, respectivamente. 
resultou em marcação superior à dos outros métodos, que não diferiram entre si. Os métodos para as raízes da camada de $0,2-0,4 \mathrm{~m}$ e para as da profundidade $>0,4 \mathrm{~m}$ também diferiram estatisticamente, e o da injeção no colmo das plantas foi superior, na média, ao método de absorção radicular, e igual ao de pulverização foliar $(\mathrm{p}>0,05)$.

Os métodos de marcação não apresentaram diferença significativa em rizoma e raízes da camada de $0,0-0,2 \mathrm{~m}$, apesar de os valores médios indicarem a nítida superioridade de marcação do método por injeção no colmo, em relação ao da pulverização foliar, que por sua vez foi superior ao da absorção radicular. Esses resultados podem ser explicados pelo alto coeficiente de variação nesses dois compartimentos da planta, principalmente o rizoma, para o qual o valor foi de $40 \%$. Essa observação é necessária, pois a marcação da fitomassa pelo método por injeção no colmo da planta foi cerca de 70 e $35 \%$ superior em abundância isotópica, comparada à absorção radicular, para rizomas e raízes da camada de $0,0-0,2 \mathrm{~m}$, respectivamente.

A diferença na marcação com ${ }^{15} \mathrm{~N}$ das raízes de profundidades distintas foi observada experimentalmente, em campo, por Faroni (2004). Essa desuniformidade na distribuição do ${ }^{15} \mathrm{~N}$ pode ter como causa o tempo entre o início da marcação e a colheita da planta, que pode ter sido insuficiente para uma distribuição uniforme do marcador. Outra explicação é que a planta pode ter uma distribuição preferencial de $\mathrm{N}$, pois as raízes mais profundas podem ser essencialmente absorvedoras de água ou solução do solo e possuem reduzida atividade metabólica e de crescimento; as raízes mais superficiais apresentam atividade metabólica na absorção de nutrientes e no crescimento de novas raízes (Casagrande, 1991).
Foram também calculados os valores de recuperação do marcador ${ }^{15} \mathrm{~N}$-uréia aplicado por parcela, e para cada um dos compartimentos estudados (Tabela 4), a fim de se avaliar em qual dos métodos houve menor perda e melhor eficiência de marcação, tendo sido encontradas diferentes recuperações nas partes da planta.

A maior recuperação total foi obtida com o método da injeção no colmo das plantas, superior à pulverização foliar e à imersão radicular em 2,75 e 4,48 vezes, respectivamente. Pela análise da recuperação em cada compartimento, o método da injeção no colmo da planta foi significativamente superior aos demais, na recuperação do marcador, tendo sido 3,51 vezes maior ao da aplicação foliar na parte aérea da planta. A maior recuperação do isótopo, quando este é injetado na planta, ocorre pelo fato de toda a solução ser aplicada no interior da planta; além disso, após a uréia sofrer hidrólise pela ação da urease, o marcador, na forma de ${ }^{15} \mathrm{NH}_{3}$ ou de ${ }^{15} \mathrm{NH}_{4}{ }^{+}$, é distribuído à planta pelo sistema de feixes vasculares (Witte et al.,2002).

Para a recuperação do marcador nos rizomas, os métodos da injeção e da aplicação foliar foram igualmente eficientes e superiores ao da imersão radicular, o qual obteve as menores recuperações, o que contrasta com as informações apresentadas por Russel \& Fillery (1996). Esse fato pode ser explicado pela absorção parcial da solução que continha o marcador pelas raízes das plantas, evidenciado pela observação de resíduos de uréia, nos frascos Ependorf, no momento da colheita do experimento.

A recuperação nas raízes foi semelhante entre os métodos; a do método de imersão radicular foi inferior ao método da injeção no colmo, somente nas raízes amostradas de 0,2-0,4 $\mathrm{m}$ de profundidade. Como o sistema radicular compõe o compartimento de maior teor

Tabela 4. Recuperação do marcador ${ }^{15} \mathrm{~N}$-uréia na aplicação foliar (F), na injeção no colmo da planta (I) e na imersão radicular (R), nos compartimentos da parte aérea, rizomas e raízes das plantas ${ }^{(1)}$.

\begin{tabular}{|c|c|c|c|c|c|c|c|c|c|}
\hline \multirow[t]{2}{*}{ Compartimento } & \multicolumn{3}{|c|}{ N-total } & \multicolumn{3}{|c|}{ NPPM } & \multicolumn{3}{|c|}{ Recuperação ${ }^{15} \mathrm{~N}$} \\
\hline & $\mathrm{F}$ & I & $\mathrm{R}$ & $\mathrm{F}$ & I & $\mathrm{R}$ & $\mathrm{F}$ & I & $\mathrm{R}$ \\
\hline & \multicolumn{6}{|c|}{----------------------------------(g por vaso)----------------------------------- } & \multicolumn{3}{|c|}{-------------------------(\%)----------------------- } \\
\hline Parte aérea & 3,42 & 5,03 & 3,89 & 0,369 & 0,247 & 0,044 & $24,0 \mathrm{~b}$ & $84,2 \mathrm{a}$ & $15,1 b$ \\
\hline Rizomas & 1,11 & 1,08 & 1,23 & 0,124 & 0,028 & 0,014 & $8,0 \mathrm{a}$ & $9,5 \mathrm{a}$ & $4,8 \mathrm{~b}$ \\
\hline \multicolumn{10}{|l|}{ Raízes } \\
\hline $0-0,2 \mathrm{~m}$ & 0,42 & 0,38 & 0,32 & 0,036 & 0,005 & 0,003 & $2,4 a$ & $1,7 \mathrm{a}$ & $1,2 \mathrm{a}$ \\
\hline $0,2-0,4 \mathrm{~m}$ & 0,18 & 0,22 & 0,17 & 0,004 & 0,001 & 0,0004 & $0,3 a b$ & $0,5 \mathrm{a}$ & $0,1 \mathrm{~b}$ \\
\hline$>0,4 \mathrm{~m}$ & 0,30 & 0,25 & 0,28 & 0,004 & 0,001 & 0,0005 & $0,3 \mathrm{a}$ & $0,3 \mathrm{a}$ & $0,2 \mathrm{a}$ \\
\hline Total & - & - & - & 0,537 & 0,282 & 0,062 & $35,0 \mathrm{~b}$ & $96,2 \mathrm{a}$ & $21,4 \mathrm{~b}$ \\
\hline
\end{tabular}

(1)Médias seguidas por letras iguais, na linha, não diferem entre si pelo teste de Tukey, a 5\% de probabilidade; NPPM: N na planta proveniente do marcador. 
de $\mathrm{N}$, o maior direcionamento do traçador aplicado deve ter sido dado a essas estruturas morfológicas, as quais também possuem papel de reserva nutricional para a rebrota (Trivelin et al., 2002).

A recuperação do marcador no solo foi baixa (menor que $0,08 \%$ ), à exceção da marcação via aplicação foliar, na camada de $0,0-0,2 \mathrm{~m}$, que foi $0,15 \%$ do ${ }^{15} \mathrm{~N}$ aplicado; não houve diferença entre os tratamentos. Essa baixa recuperação é consequiência da passagem de raízes finas e radicelas no processo de peneiramento (Noordwijk \& Floris, 1979; Faroni \& Trivelin, 2006), o que mostra que o método não marcou efetivamente o solo, como inicialmente proposto na metodologia do trabalho.

\section{Conclusões}

1. A injeção de solução de uréia marcada com ${ }^{15} \mathrm{~N}$ no colmo de plantas de cana-de-açúcar é o método de mais fácil uso, de mais efetiva marcação $\operatorname{com}{ }^{15} \mathrm{~N}$ da fitomassa e o que apresenta maior recuperação do marcador.

2. O método da pulverização foliar é comparável ao de injeção, para a marcação em ${ }^{15} \mathrm{~N}$ e a recuperação do marcador no sistema radicular.

3. Não é recomendável o uso do método por absorção radicular na marcação de fitomassa (parte aérea e sistema radicular) de cana-de-açúcar.

\section{Agradecimentos}

À Fapesp pelo financiamento do trabalho; à APTA Pólo Regional Centro Sul, pela cessão da área para a realização do experimento.

\section{Referências}

AMBROSANO, E.J.; TRIVELIN, P.C.O.; CANTARELLA, H.; ROSSETTO, R.; MURAOKA, T.; BENDASSOLLI, J.A.; AMBROSANO, G.M.B.; TAMISO, L.G.; VIEIRA, F.C.; PRADA NETO, I. Nitrogen-15 labeling of Crotalaria juncea green manure. Scientia Agricola, v.60, p.181-184, 2003.

BARRIE, A.; PROSSER, S.J. Automated analysis of light-element stable isotopes by isotope ratio mass spectrometry. In: BOUTTON, T.W.; YAMSAHI, S. (Ed.). Mass spectrometry of soils. New York: Marcel Dekker, 1996. p.1-46.

CASAGRANDE, A.A. Tópicos de morfologia e fisiologia da cana-de-açúcar. Jaboticabal: Funep, 1991. 157p.

EMBRAPA. Centro Nacional de Pesquisa de Solos. Sistema brasileiro de classificação de solos. Rio de Janeiro: Embrapa-SPI, 1999. 412p.

ESPIRONELLO, A.; RAIJ, B. van; PENATTI, C.P.; CANTARELLA, H.; MORELLI, J.L.; ORLANDO FILHO, J.;
LANDELL, M.G.A.; ROSSETTO, R. Cana-de-açúcar. In: RAIJ, B. van; CANTARELLA, H.; QUAGGIO, J.A.; FURLANI, A.M.C. (Ed.). Recomendações de adubação e calagem para o Estado de São Paulo. Campinas: Fundação IAC, 1996. p.237-239. (Boletim, 100).

FARONI, C.E. Sistema radicular de cana-de-açúcar e identificação de raízes metabolicamente ativas. 2004. 68p. Dissertação (Mestrado) - Universidade de São Paulo, Piracicaba.

FARONI, C.E.; TRIVELIN, P.C.O. Quantificação de raízes metabolicamente ativas de cana-de-açúcar. Pesquisa Agropecuária Brasileira, v.41, p.1007-1013, 2006.

GAVA; G.J.C.; TRIVELIN, P.C.O.; VITTI. A.C.; OLVEIRA, M.W. Urea and sugarcane straw nitrogen balance in a soil-sugarcane crop system. Pesquisa Agropecuária Brasileira, v.40, p.689-695, 2005.

HUMBERT, R.P. Fertilization by aircraft in the Hawaiian sugar industry. The Hawaiian Planter's Record, v.55, p.301-311, 1960.

MALAVOLTA, E. Fertilizing for high yield sugarcane. Basel: IPI, 1994. 104p. (Bulletin, 14).

McNEILL, A.M.; ZHU, C.; FILLERY, R.P. Use of in situ ${ }^{15} \mathrm{~N}$-labelling to estimate the total below-ground nitrogen of pasture legumes in intact soil-plant systems. Australian Journal of Agriculture Research, v.48, p.295-304, 1997.

NOORDWIJK, M. van; FLORIS, J. Loss of dry weight during washing and storage of root samples. Plant and Soil, v.53, p.239$243,1979$.

RAIJ, B. van. Fertilidade do solo e adubação. Piracicaba: Editora Agronômica Ceres, 1991. 343p.

RUSSEL, C.A.; FILLERY, I.R.P. In situ ${ }^{15} \mathrm{~N}$ labeling of lupin belowground biomass. Australian Journal of Agricultural Research, v.47, p.1035-1046, 1996.

THORNE, G. Nutrient uptake from leaf sprays by crops. Field Crops Abstracts, v.8, p.147-151, 1955.

TRIVELIN, P.C.O. Utilização do nitrogênio pela cana-de-açúcar: três casos estudados com o uso do traçador ${ }^{15}$ N. 2000. 143p. Tese (Livre-docência) - Universidade de São Paulo, Piracicaba.

TRIVELIN, P.C.O.; CARVALHO, J.G.; SILVA, A.Q.; PRIMAVESI, A.C.P.A.; CAMACHO, E.; EIMORI, I.E.; GUILHERME, M.R. Adubação foliar de cana-de-açúcar (Saccharum spp.). Absorção e translocação de N-uréia. Energia Nuclear e Agricultura, v.9, p.5265, 1988.

TRIVELIN, P.C.O.; VITTI, A.C.; OLIVEIRA, M.W.; GAVA, G.J.C.; SARRIÉS, G.A. Utilização de nitrogênio e produtividade da cana-de-açúcar (cana-planta) em solo arenoso com incorporação de resíduos da cultura. Revista Brasileira de Ciência do Solo, v.26, p.636-646, 2002.

VASILAS, B.L.; LEGG, J.O.; WOLF, D.C. Foliar fertilization of soybeans: absorption and translocation of ${ }^{15} \mathrm{~N}$-labelled urea. Agronomy Journal, v.72, p.271-275, 1980.

WITTE, C.P.; TILLER, S.A.; TAYLOR, M.A.; DAVIES, H.V. Leaf urea metabolism in potato: urease activity profile and patterns of 
recovery and distribution of ${ }^{15} \mathrm{~N}$ after foliar urea application in wildtipe and urease-antisense transgenics. Plant Physiology, v.128, p.1129-1136, 2002.

WITTWER, S.H.; BUKOVAC, M.J.; TUKEY, H.B. Advances in foliar feeding of plant nutrients. In: McVICKAR, M.H.; BRIDGER, G.L.; NELSON, L.B. (Ed.). Fertilizer technology and usage. Madison: American Society of Agronomy, 1963. p.429-455.
WITTWER, S.H.; TEUBNER, F.G. Foliar absorption of mineral nutrients. Annual Review of Plant Physiology, v.10, p.13-32, 1959.

ZEBARTH; B.J.; ALDER, V.; SHEARD, R.W. In situ labelling of legume residues with a foliar application of a ${ }^{15} \mathrm{~N}$-enriched urea solution. Communications in Soil Science and Plant Analysis, v.22, p.437-447, 1991.

Recebido em 22 de janeiro de 2007 e aprovado em 25 de maio de 2007 